\title{
Energy-Optimal Probabilistic Base Station Sleeping under a Separation Network Architecture
}

\author{
Shan Zhang, Jian Wu, Jie Gong, Sheng Zhou, Zhisheng Niu \\ Tsinghua National Laboratory for Information Science and Technology, \\ Dept. of Electronic Engineering, Tsinghua Univ., Beijing, 100084, P.R. China \\ Email: \{zhangshan11,wujian09,gongj13\}@mails.tsinghua.edu.cn, \{sheng.zhou,niuzhs\}@tsinghua.edu.cn
}

\begin{abstract}
To further improve energy efficiency from the view of the whole network, a separation architecture has been proposed, where the control plane and data plane are separated and implemented by different base stations. Under this architecture, the data base stations (DBS) can be turned off adaptively according to the traffic load while signaling base stations (SBS) provide the guarantee of coverage. A key issue of this architecture is the design of effective BS sleeping mechanisms, which should guarantee the quality of service $(\mathrm{QoS})$ and minimize network power consumption. In this paper, a probabilistic DBS sleeping mechanism is proposed and optimized under the separation architecture. Users within the sleeping DBSs are offloaded to SBSs for QoS guarantee. An optimization problem is formulated, where the sleeping probability and spectrum resource allocation are jointly optimized to minimize network power consumption. The optimal BS sleeping scheme is found to be threshold-based. When the ratio of sleeping DBSs is below a certain threshold which depends on the traffic load, the lightly-loaded DBSs should be turned off first; otherwise, only the heavily loaded DBSs go into sleep. Numerical results show nearly $30 \%$ energy can be saved under a typical daily traffic profile, and there exists a tradeoff between energy saving and network capacity.
\end{abstract}

\section{INTRODUCTION}

Multi-tier heterogeneous networks (HetNet) is promising to boost network capacity, but will also cause huge power consumption for the densely deployed base stations (BS) [1] [2]. To solve this problem, a new architecture based on HetNet called hyper-cellular network has been proposed [3]-[5]. Under the hyper-cellular network architecture, control and signaling functions are implemented by signaling base stations (SBS), while data base stations (DBS) are only responsible for data transmission. Therefore, DBSs can be turned off without compromising coverage.

The quality of service (QoS) of the sleeping BSs also needs to be guaranteed. Therefore, before a BS goes into sleep, its users need to be offloaded to other active BSs to continue service [6]-[8]. Heuristic BS sleeping algorithms were proposed in [6] and [7], where the lightly-loaded BSs are turned off first. Besides, HetNets were considered in [8],

This work is sponsored in part by the National Science Foundation of China (NSFC) under grant No. 61201191 and No. 61401250, the National Basic Research Program of China (973 Program: 2012CB316001), the Creative Research Groups of NSFC under grant No. 61321061, and Hitachi R\&D Headquarter. where two sleeping mechanism were proposed and analyzed theoretically. To guarantee the QoS, the users of sleeping BSs are offloaded to active neighboring BSs.

In fact, these BS sleeping mechanisms are insufficient. Under the traditional network architecture, the users can only be offloaded to the neighboring BSs. As the performance of neighboring cells is coupled, the optimal algorithm of BS sleeping has high complexity, while the heuristic algorithms are not efficient and may cause outage problems. Besides, the coverage of the sleeping BSs can not be well guaranteed by neighboring BSs in real networks for the limitation of physical systems parameters, e.g. transmit power and antenna tilt. As a result, these BS sleeping algorithms are difficult to be implemented in practice.

Under the separation architecture, DBS sleeping can be easily conducted as the coverage of the network can be well guaranteed by the SBSs. Therefore, a key design issue is the DBS sleeping scheme. In this paper, we explore the energy-optimal probabilistic DBS sleeping mechanism under the separation network architecture, where each DBS goes into sleep probabilistically based on its traffic load. Meanwhile, users of the sleeping DBSs are offloaded to the SBS-tier (inter-tier offloading). In addition, dynamic spectrum resource management is also considered. To solve this problem, its dual problem is analyzed, where the network capacity is maximized for the given network power consumption. The optimal operation of the subproblems is as follows:

1) $\bar{p}_{\text {off }}<\theta_{\text {off }}$ : the lightly-loaded DBSs should be turned off first;

2) $\bar{p}_{\text {off }}>\theta_{\text {off }}$ : the heavily-loaded DBSs should go into sleep;

3) $\bar{p}_{\text {off }}=\theta_{\text {off }}$ : all sleeping schemes have the same performance.

Here, $\bar{p}_{\text {off }}$ is the ratio of DBSs in sleep mode which determines the given network power consumption, and $\theta_{\text {off }}$ is a threshold. Numerical results show that nearly 30\% energy can be saved through the optimal solution under a typical daily traffic profile.

The rest of the paper is organized as follows: Sec. II describes the system model and DBS sleeping problem. Sec. III introduces problem formulation. Then, based on 


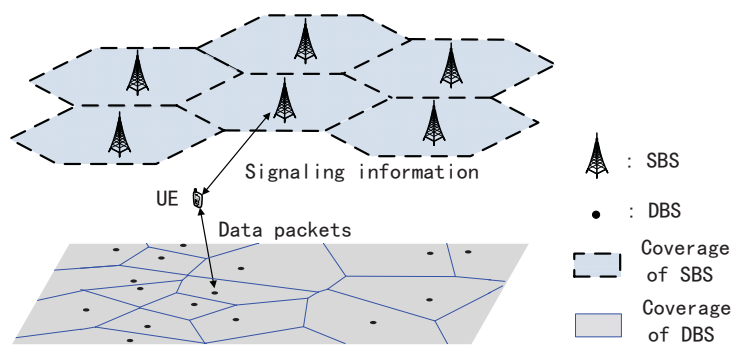

Fig. 1: Separation architecture

theoretical analysis, the optimal BS sleeping scheme is obtained in Sec. IV. Numerical results are shown in Sec. V. Sec. VI concludes this paper.

\section{System Model and Probabilistic DBS Sleeping}

\section{A. System Model}

Fig. 1 shows the separation architecture[3]-[5]. Two tiers of BSs are deployed and the system model is as follows:

1) Signaling Base Stations (SBS) The SBSs are usually regularly deployed and have larger coverage area. They are mainly responsible for control and signaling information, and guarantees the coverage of the whole network.

2) Data Base Stations (DBS) The DBSs, which are responsible for data transmission, are mainly pico BSs or femto BSs with much smaller cells. Besides, DBSs are more randomly deployed, whose distribution can be modeled as homogeneous Poisson Point Processes (PPP).

3) User Equipments (UE) Assume UEs are uniformly distributed independently of the location of BSs. Furthermore, the distribution of UEs is also modeled as a homogeneous PPP. Each UE should maintain two connections simultaneously: one with the SBS-tier for functions like paging and broadcasting, and one with DBS-tier for data transmission (as shown in Fig. 1). Besides, maximal SINR scheme is adopted for the intra-tier UE-BS association.

4) Bandwidth Allocation (BA) The system bandwidth is divided into two parts: signaling bandwidth for signaling and control service, and data bandwidth for data transmission. Signaling bandwidth is only allocated to the SBSs while data bandwidth is initially allocated to the DBSs. During low traffic hours, the redundant bandwidth of DBSs will be released and re-allocated to the SBSs for traffic offloading such that more DBSs can go into sleep. The system spectrum resource partitioning is demonstrated in Fig. 2. We focus on the data bandwidth management in this paper, and the partitioning between signaling bandwidth and data bandwidth has been discussed in our previous work [9]. Denote the bandwidth of the data bandwidth as $W$, which is shared between SBSs and DBSs when traffic offloading is conducted as shown in Fig. 2. The

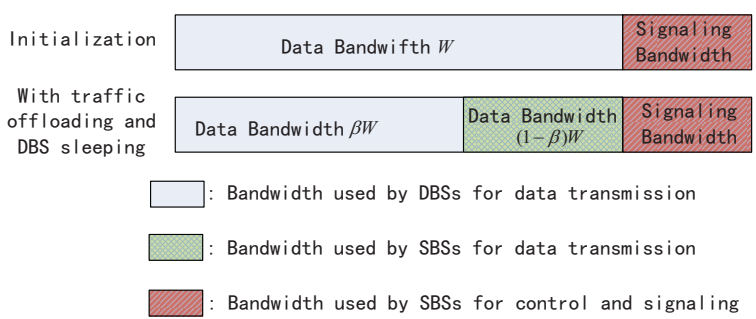

Fig. 2: System spectrum resource partitioning

partition ratio $\beta$ is to be optimized. Besides, assume the intra-tier spectrum reuse factor is 1, i.e., the bandwidth of each DBS is $\beta W$ and the bandwidth of each SBS for data transmission is $(1-\beta) W$.

\section{B. Link Layer Model}

Denote by $P_{\mathrm{td}}$ the transmit power of DBSs, then the received SINR of $\mathrm{UE}_{u}$ associated with $\mathrm{DBS}_{i}$ is given by

$$
\gamma_{\mathrm{d}}=\frac{P_{\mathrm{td}} G_{i u}}{\sum_{j \in \mathcal{D}_{\mathrm{A}}, j \neq i} P_{\mathrm{td}} G_{j u}+\sigma^{2}},
$$

where $\sigma^{2}$ is the noise power, $\mathcal{D}_{\mathrm{A}}$ is the set of active DBSs. $G_{i u}$ is the channel gain between $\mathrm{UE}_{u}$ and $\mathrm{DBS}_{i}$ given by

$$
G_{i u}=d_{i u}^{-\alpha} h_{i u}
$$

$d_{i u}$ is the distance between $\mathrm{UE}_{u}$ and $\mathrm{DBS}_{i}, \alpha$ is the path loss factor ( $2<\alpha \leq 4$ for cellular networks), and $h_{i u}$ is a random variable that incorporates the effect of fading. Thus, the achievable rate of $\mathrm{UE}_{u}$ is given by the Shannon formula for a AWGN channel:

$$
r_{\mathrm{d}}=\frac{\beta W}{N_{\mathrm{d}}} \log _{2}\left(1+\gamma_{\mathrm{d}}\right),
$$

where $W$ is the total bandwidth for data transmission, $\beta$ is the proportion of bandwidth used by DBSs $(\beta=1$ without considering traffic offloading), $N_{\mathrm{d}}$ is a random variable denoting the number of UEs associated with $\mathrm{DBS}_{i}$.

Similarly, the achievable rate of $\mathrm{UE}_{u}$ offloaded to $\mathrm{SBS}_{k}$ is given by:

$$
r_{\mathrm{s}}=\frac{(1-\beta) W}{N_{\mathrm{s}}} \log _{2}\left(1+\gamma_{\mathrm{s}}\right)
$$

where $N_{\mathrm{s}}$ is the number of UEs offloaded to $\mathrm{SBS}_{k}$ for data service,

$$
\gamma_{\mathrm{s}}=\frac{P_{\mathrm{ts}} G_{k u}}{\sum_{l \in \mathcal{S}, l \neq k} P_{\mathrm{ts}} G_{l u}+\sigma^{2}},
$$

$P_{\mathrm{ts}}$ is the transmit power of SBSs and $\mathcal{S}$ is the set of all SBSs.

\section{Load-Aware DBS sleeping Scheme}

We consider a load-aware probabilistic DBS sleeping scheme. $\mathrm{DBS}_{i}$ goes into sleep with probability $p_{\text {off }}\left(n_{i}\right)$, where $n_{i}$ is the number of UEs served by $\mathrm{DBS}_{i}$. By 
designing $p_{\text {off }}(n)$, a variety of DBS sleeping schemes can be denoted. For example, if $p_{\text {off }}(n)$ is given by

$$
p_{\text {off }}(n)= \begin{cases}1, & n \leq N^{*} \\ 0, & n>N^{*}\end{cases}
$$

only the lightly loaded DBSs are turned off and $N^{*}$ is the threshold [10]. Furthermore, if $p_{\text {off }}(n)=\eta_{p}\left(0 \leq \eta_{p} \leq 1\right)$, all DBSs are turned off with equivalent probability $\eta_{p}$ regardless of their traffic load.

\section{Problem Formulation AND SOlution}

\section{A. Problem Formulation}

As the DBSs in sleep mode consume much smaller power than in the active mode, DBSs should be turned off as many as possible to minimize network power consumption. Therefore the problem can be formulated as follows:

$$
\begin{aligned}
\max _{\beta, p_{\text {off }}(n)} & \sum_{n=0}^{\infty} p_{\text {off }}(n) f_{N_{\mathrm{d}}}(n) \\
\text { s.t. } & \mathbb{E}\left\{r_{\mathrm{d}}\right\} \geq U_{\mathrm{d}} \\
& \mathbb{E}\left\{r_{\mathrm{s}}\right\} \geq U_{\mathrm{s}},
\end{aligned}
$$

where $f_{N_{\mathrm{d}}}$ is the probability distribution function of the number of UEs per DBS cell, $U_{s}$ (or $U_{d}$ ) is the required data rate of SBS (or DBS) UEs, and $r_{\mathrm{s}}$ (or $r_{\mathrm{d}}$ ) is the data rate of a typical SBS (or DBS) UE. The objective function of this problem is the ratio of DBSs in sleep mode (or the average probability that a DBS can go into sleep), and the two constraints guarantee the QoS performance. Note that the ratio of sleeping DBSs $\bar{p}_{\text {off }}$ corresponds to the network power consumption if power control is neglected. Besides, the two constraints offers the minimal network capacity. Therefore, the physical meaning of problem (7) is to find the optimal DBS sleeping scheme which can minimize network power consumption under the given constraint of network capacity.

The optimal solution of of problem (7) can be obtained by the exhaustive search. Consider the following problem:

$$
\begin{aligned}
\max _{\beta, p_{\text {off }}(n)} & \mathbb{E}\left\{r_{\mathrm{s}}\right\} \\
\text { s.t. } & \mathbb{E}\left\{r_{\mathrm{d}}\right\} \geq U_{\mathrm{d}} \\
& \sum_{n=0}^{\infty} p_{\text {off }}(n) f_{N_{\mathrm{d}}}(n)=\bar{p}_{\text {off }},
\end{aligned}
$$

where the ratio of sleeping DBSs $\bar{p}_{\text {off }}\left(0 \leq \bar{p}_{\text {off }} \leq 1\right)$ is given as a parameter. Problem (7) and problem (8) are dual problems. The physical meaning of problem (7) is to minimize power consumption under the network capacity constraint, whereas problem (8) is to maximize the network capacity when the power is limited.

If the optimal value of (8) is no smaller than $U_{\mathrm{s}}$, the optimal solution of (8) is also feasible for problem (7) as the QoS requirement of DBSs and SBSs can be satisfied simultaneously. In this case, the optimal solution of (7) will be no smaller than the $\bar{p}_{\text {off }}$ of (7). However, problem (8) may be infeasible if $\bar{p}_{\text {off }}$ is too large to meet the QoS requirements of UEs, and the optimal solution of (7) should be smaller than $\bar{p}_{\text {off }}$. Therefore, our problem becomes to find the maximal $\bar{p}_{\text {off }}$ of problem (8), under which the optimal solution of (8) is feasible for problem (7).

\section{UE PERFORMANCE ANALYSIS}

\section{A. Average Data Rate of Offloaded UES}

Based on Eq. (3), the average achievable rate for a typical DBS UE is given by

$$
\begin{aligned}
\mathbb{E}\left\{r_{\mathrm{d}}\right\} & =\mathbb{E}\left\{\frac{\beta W}{N_{\mathrm{d}}} \log _{2}\left(1+\gamma_{\mathrm{d}}\right)\right\} \\
& \approx \beta W \frac{\mathbb{E}\left\{\log _{2}\left(1+\gamma_{\mathrm{d}}\right)\right\}}{\mathbb{E}\left\{N_{\mathrm{d}}\right\}} .
\end{aligned}
$$

Substituting (9) into the first constraint in (8), we have

$$
\beta \geq \frac{U_{\mathrm{d}} \mathbb{E}\left\{N_{\mathrm{d}}\right\}}{W \mathbb{E}\left\{\log _{2}\left(1+\gamma_{\mathrm{d}}\right)\right\}} .
$$

The average data rate of the offloaded UE is given by

$$
\begin{aligned}
\mathbb{E}\left\{r_{\mathrm{s}}\right\} & \approx(1-\beta) W \frac{\mathbb{E}\left\{\log _{2}\left(1+\gamma_{\mathrm{s}}\right)\right\}}{\mathbb{E}\left\{N_{\mathrm{s}}\right\}} \\
& \leq\left(W-\frac{U_{\mathrm{d}} \mathbb{E}\left\{N_{\mathrm{d}}\right\}}{\mathbb{E}\left\{\log _{2}\left(1+\gamma_{\mathrm{d}}\right)\right\}}\right) \frac{\mathbb{E}\left\{\log _{2}\left(1+\gamma_{\mathrm{s}}\right)\right\}}{\mathbb{E}\left\{N_{\mathrm{s}}\right\}} .
\end{aligned}
$$

Note that $\mathbb{E}\left\{r_{\mathrm{s}}\right\}$ can be optimized only when the equality of (10) and (11) holds. Thus we take the equality of (10) and (11) in the rest of this paper.

After the DBSs go into sleep with probability $p_{\text {off }}(n)$, the average number of UEs offloaded to each SBS $\mathbb{E}\left\{N_{\mathrm{s}}\right\}$ is given by

$$
\mathbb{E}\left\{N_{\mathrm{s}}\right\}=\frac{\rho_{\mathrm{d}}}{\rho_{\mathrm{s}}} \sum_{n=1}^{\infty} p_{\text {off }}(n) f_{N_{\mathrm{d}}}(n) n,
$$

and the average number of UEs in each active DBS $\mathbb{E}\left\{N_{\mathrm{d}}\right\}$ is given by

$$
\begin{aligned}
\mathbb{E}\left\{N_{\mathrm{d}}\right\} & =\sum_{n=1}^{\infty} \frac{\left(1-p_{\text {off }}(n)\right) f_{N_{\mathrm{d}}}(n) n}{1-\bar{p}_{\text {off }}} \\
& =\frac{1}{1-\bar{p}_{\text {off }}}\left(\mathbb{E}\left\{N_{0 \mathrm{~d}}\right\}-\frac{\rho_{\mathrm{s}}}{\rho_{\mathrm{d}}} \mathbb{E}\left\{N_{\mathrm{s}}\right\}\right),
\end{aligned}
$$

where $\rho_{\mathrm{d}}$ and $\rho_{\mathrm{s}}$ are the density of DBSs and SBSs respectively, $\mathbb{E}\left\{N_{0 \mathrm{~d}}\right\}$ is the average number of UEs in each DBS before DBS sleeping and $f_{N_{\mathrm{d}}}(n)$ is the probability distribution function of $N_{\mathrm{d}}$. Based on the theory of stochastic geometry, $N_{\mathrm{d}}$ follows Poisson distribution and we have

$$
f_{N_{\mathrm{d}}}(n)=\frac{\left(\frac{\lambda}{\rho_{\mathrm{d}}}\right)^{\mathrm{n}}}{n !} \exp \left(-\frac{\lambda}{\rho_{\mathrm{d}}}\right),
$$

where $\lambda$ is the density of UEs.

Notice that $\mathbb{E}\left\{N_{0 \mathrm{~d}}\right\}$ is independent of the sleeping scheme $p_{\text {off }}(n)$. Substituting (12) into (11), we have

$$
\begin{aligned}
& \mathbb{E}\left\{r_{\mathrm{s}}\right\}=\left(W-\frac{U_{\mathrm{d}} \mathbb{E}\left\{N_{\text {Od }}\right\}}{\mathbb{E}\left\{\log _{2}\left(1+\gamma_{\mathrm{d}}\right)\right\}\left(1-\bar{p}_{\text {off }}\right)}\right) \\
& \cdot \frac{\mathbb{E}\left\{\log _{2}\left(1+\gamma_{\mathrm{s}}\right)\right\}}{\mathbb{E}\left\{N_{\mathrm{s}}\right\}}+\frac{\rho_{\mathrm{s}}}{\rho_{\mathrm{d}}} \frac{U_{\mathrm{d}} \mathbb{E}\left\{\log _{2}\left(1+\gamma_{\mathrm{s}}\right)\right\}}{\mathbb{E}\left\{\log _{2}\left(1+\gamma_{\mathrm{d}}\right)\right\}\left(1-\bar{p}_{\text {off }}\right)} .
\end{aligned}
$$




\section{B. Optimal DBS Sleeping Scheme}

The average spectrum efficiency of the offloaded SBS UEs $\mathbb{E}\left\{\log _{2}\left(1+\gamma_{\mathrm{s}}\right)\right\}$ is independent of the DBS sleeping schemes, as SBSs and DBSs use orthogonal spectrum. In addition, the average spectrum efficiency of DBS UEs $\mathbb{E}\left\{\log _{2}\left(1+\gamma_{\mathrm{d}}\right)\right\}$ depends on the ratio of sleeping DBSs $\bar{p}_{\text {off }}$ rather than the sleeping scheme $p_{\text {off }}(n)$. As $\bar{p}_{\text {off }}$ in problem (8) is a parameter, the only different part of (15) under different sleeping schemes $p_{\text {off }}(n)$ is $\mathbb{E}\left\{N_{\mathrm{s}}\right\}$.

Denote

$$
\theta_{\text {off }}=1-\frac{U_{\mathrm{d}} \mathbb{E}\left\{N_{0 \mathrm{~d}}\right\}}{W \mathbb{E}\left\{\log _{2}\left(1+\gamma_{\mathrm{d}}\right)\right\}} .
$$

The optimal DBS sleeping scheme is as follows.

1) $\bar{p}_{\text {off }}<\theta_{\text {off }}: \mathbb{E}\left\{r_{\mathrm{s}}\right\}$ decreases with $\mathbb{E}\left\{N_{\mathrm{s}}\right\}$, and thus $\mathbb{E}\left\{N_{\mathrm{s}}\right\}$ needs to be minimized to achieve the optimal value of problem (8). In this case, the optimal sleeping scheme is given by

$$
\begin{cases}p_{\text {off }}(n)=1, & n<N^{*} \\ p_{\text {off }}(n)=0, & n>N^{*} \\ p_{\text {off }}(n)=p_{\text {off }}^{\prime}, & n=N^{*}\end{cases}
$$

where $N^{*}$ and $0 \leq p_{\text {off }}^{\prime}<1$ satisfies

$$
\sum_{n=0}^{N^{*}-1} f_{N_{\mathrm{d}}}(n)+p_{\text {off }}^{\prime} f_{N_{\mathrm{d}}}\left(N^{*}\right)=\bar{p}_{\text {off }} .
$$

This scheme is named as "lightly-loaded off scheme", as only the DBSs whose traffic load is below $N^{*}$ are turned off ${ }^{1}$.

2) $\bar{p}_{\text {off }}>\theta_{\text {off }}: \mathbb{E}\left\{r_{\mathrm{s}}\right\}$ increases with $\mathbb{E}\left\{N_{\mathrm{s}}\right\}$ and the optimal DBS sleeping scheme is given by

$$
\begin{cases}p_{\text {off }}(n)=0, & n<N^{*} \\ p_{\text {off }}(n)=1, & n>N^{*} \\ p_{\text {off }}(n)=p_{\text {off }}^{\prime}, & n=N^{*}\end{cases}
$$

where $N^{*}$ and $0 \leq p_{\text {off }}^{\prime}<1$ satisfies

$$
\sum_{n=N^{*}+1}^{\infty} f_{N_{\mathrm{d}}}(n)+p_{\text {off }}^{\prime} f_{N_{\mathrm{d}}}\left(N^{*}\right)+f_{N_{\mathrm{d}}}(0)=\bar{p}_{\text {off }}{ }^{2} .
$$

In this case, the heavily-loaded DBSs go into sleep first ("heavily-loaded off scheme").

3) $\bar{p}_{\text {off }}=\theta_{\text {off }}$ : Different DBS sleeping schemes have the same performance.

To summarize, the lightly-loaded BSs should be turned off first when the sleeping probability $\bar{p}_{\text {off }}$ is low, while the most heavily-loaded ones should go into sleep when the sleeping probability is high.

\section{Insights of Optimal Scheme}

As $\bar{p}_{\text {off }}$ corresponds to the power consumption of the network, the physical meaning of the optimization problem (8) is to maximize network capacity for the given power consumption. Besides, the network capacity depends on the spectrum efficiency of the network, and thus, the spectrum efficiency needs to be maximized.

\footnotetext{
${ }^{1} N^{*}$ and $p_{\text {off }}^{\prime}$ can be obtained by substituting Eq. (14) into Eq. (18)

${ }^{2}$ The idle DBSs which have no users are always turned off.
}

TABLE I: Simulation Parameters

\begin{tabular}{||c|c||c|c||}
\hline Parameter & Value & Parameter & Value \\
\hline$\rho_{\mathrm{d}}$ & $100 / \mathrm{km}^{2}$ & $\rho_{\mathrm{s}}$ & $11 / \mathrm{km}^{2}$ \\
$\alpha$ & 3.5 & $W$ & $10 \mathrm{MHz}$ \\
$U_{\mathrm{d}}$ & $0.5 \mathrm{Mbps}$ & $\sigma^{2}$ & $-104 \mathrm{dBm}$ \\
$P_{\mathrm{ts}}$ & $10 \mathrm{~W}$ & $P_{\mathrm{td}}$ & $1 \mathrm{~W}$ \\
$P_{0 \mathrm{~s}}$ & $56 \mathrm{~W}$ & $P_{0 \mathrm{~d}}$ & $10 \mathrm{~W}$ \\
$\Delta_{\mathrm{S}}$ & 4 & $\Delta_{\mathrm{d}}$ & 4 \\
\hline
\end{tabular}

The essential difference of all the sleeping schemes is the different amount of bandwidth allocated to DBSs and SBSs (Eq. 10). For example, the lightly-loaded off scheme allocates the maximal bandwidth to DBS-tier among all schemes (i.e., maximal $\beta$ ), while the heavily-loaded off scheme maximized bandwidth to each SBS (i.e., minimal $\beta$ ). Therefore, when the spectrum efficiency of the DBS-tier is higher, the lightlyloaded off scheme is optimal. Otherwise, the heavily-loaded off scheme performs best.

On the other hand, the relationship between the spectrum efficiency of the two tiers depends on $\bar{p}_{\text {off }}$. When $\bar{p}_{\text {off }}$ is small, the density of active DBSs is high and DBS-tier has much higher spectrum efficiency than the SBS-tier. Besides, the spatial spectrum efficiency of DBS-tier decreases with $\bar{p}_{\text {off }}$ while the spectrum efficiency of the SBSs remains the same. When $\bar{p}_{\text {off }}$ goes to 1 , the density of the DBSs goes to zero and the spectrum efficiency of the DBS-tier goes to zero. As a result, the lightly-loaded off scheme is optimal for low $\bar{p}_{\text {off }}$ while the heavily-loaded off scheme should be conducted when $\bar{p}_{\text {off }}$ is high. Hence, the optimal solution is reasonable.

Furthermore, the threshold $\theta_{\text {off }}$ decreases with traffic load according to Eq. (16) (as $N_{0 \mathrm{~d}}$ increases with traffic load). The reason is that more UEs will be offloaded to the SBSs when the traffic load is high. In this case, the bandwidth allocated to the SBSs quickly becomes the bottleneck as the sleeping probability increases, and heavily-loaded off scheme can mitigate this problem.

\section{NumericAl Results}

In this section, we evaluate the performance of the optimal DBS sleeping scheme for energy saving.

\section{A. Evaluation of Optimal Scheme}

Fig. 3 compares the lightly-loaded off and heavily-loaded off schemes through simulation under different $\bar{p}_{\text {off }}$ and UE density $\lambda$. Besides, these two schemes are also compared with a randomly sleeping scheme, where each DBS goes into sleep with probability $\bar{p}_{\text {off }}$ regardless of its traffic load (as shown in Fig. 4). The parameters are listed in Table I.

In Fig. $3(\mathrm{a}), W_{\mathrm{s}}^{\mathrm{l}}$ and $W_{\mathrm{s}}^{\mathrm{h}}$ are the bandwidth allocated to each SBS under the lightly-loaded off and the heavily-loaded off schemes respectively, and the z-axis shows the ratio of these two values. Obviously, lightly-loaded off scheme allocates less bandwidth to the SBSs $\left(W_{\mathrm{s}}^{\mathrm{l}} / W_{\mathrm{s}}^{\mathrm{h}}<1\right)$. In addition, the ratio of average number of UEs per SBS $\left(N_{\mathrm{s}}^{\mathrm{l}} / N_{\mathrm{s}}^{\mathrm{h}}\right)$ is shown in Fig. $3(\mathrm{~b})$, where $N_{\mathrm{s}}^{\mathrm{l}}\left(\right.$ and $\left.N_{\mathrm{s}}^{\mathrm{h}}\right)$ is the 


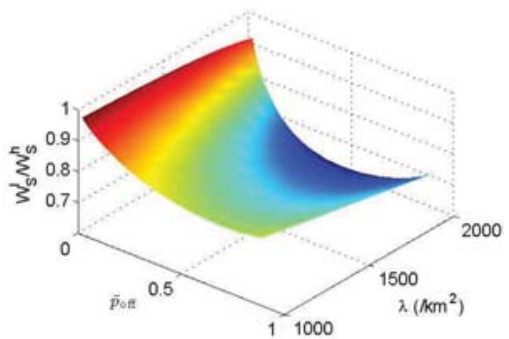

(a) Ratio of bandwidth for SBSs

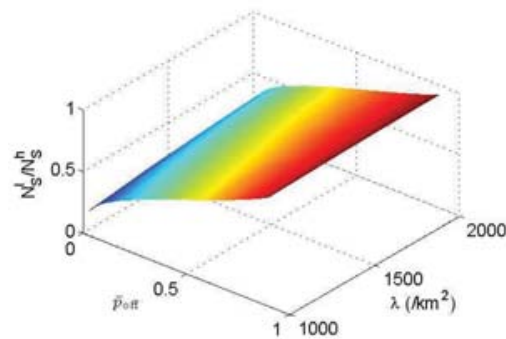

(b) Ratio of average number of UEs per SBS

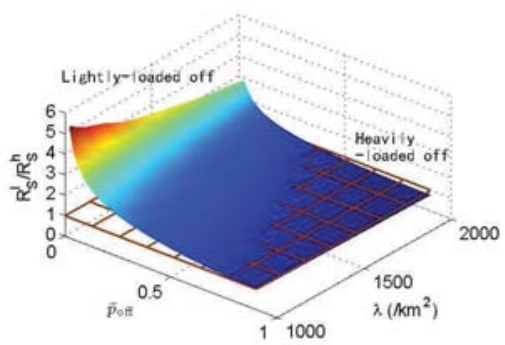

(c) Ratio of average rate of SBS UEs

Fig. 3: Comparison between two schemes

average number of UEs in each SBS under the lightly-loaded (and heavily-loaded) off scheme. Fewer UEs are offloaded to the SBSs under the lightly-loaded off scheme, especially when the ratio of sleeping DBSs and the traffic load is low. By comparing Fig. 3 (a) and Fig. 3 (b), we can conclude that the advantage of the lightly-loaded off scheme degrades as the ratio of sleeping DBSs and traffic load increase, as a result of lower density of DBSs. The comparison of rate of SBS UEs is shown in Fig. 3 (c), where the mesh plane is $z=1 . R_{\mathrm{s}}^{\mathrm{l}}$ (and $R_{\mathrm{s}}^{\mathrm{h}}$ ) is the average rate of SBS UEs under the lightly-loaded (heavily-loaded) off scheme. Fig. 3 (c) indicates which scheme is better. In the region where the surface $R_{\mathrm{s}}^{\mathrm{l}} / R_{\mathrm{S}}^{\mathrm{h}}$ is above the mesh plane, the lightly-loaded off scheme provides higher rate for SBS UEs. The heavily-loaded off scheme performs better than the lightly-loaded off scheme in the region where the surface is below the mesh plane. In addition, the two schemes achieve the same data rate in the intersection. Note that the results are consistent with our analysis in Sec. IV.

As shown in Fig. 4, the performance of the randomly sleeping scheme is always between these two schemes, which means the optimal solution is better. Fig. 4 (a) demonstrates that the threshold $\theta_{\text {off }}$ is about 0.8 when UE density is $1000 / \mathrm{km}^{2}$ (lightly-loaded). When $\lambda=2500 / \mathrm{km}^{2}$, the network is overloaded and the heavily-loaded off scheme always shows best performance as shown in Fig. 4 (b). Surprisingly, the maximal achievable rate of the offloaded SBS UEs increases when more DBSs are turned off for all schemes. This is because the interference between DBSs is lightened when more DBSs are turned off, which results in better channel condition of DBS UEs. Therefore, the DBS UEs needs less bandwidth and more bandwidth can be allocated to the SBSs. Thus, the capacity of the offloaded UEs can be improved.

Apparently, the increase of the solid lines is against the tradeoff between spectrum efficiency and energy efficiency. In fact, the average maximal rate of all UEs (including both DBS and SBS UEs) decreases when more DBSs are turned off for all schemes (shown as the dashed lines), as more UEs are offloaded to the SBSs and served at a much lower rate ${ }^{3}$.

${ }^{3} U_{\mathrm{d}}=0.5 \mathrm{Mbps}$ while the maximal $U_{\mathrm{s}}$ is less than $0.1 \mathrm{Mbps}$ in Fig. $4 \mathrm{~b}(\mathrm{~b})$.

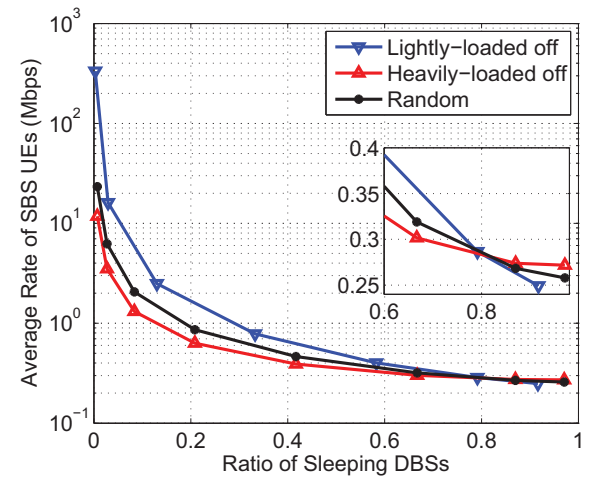

(a) $\lambda=1000 / \mathrm{km}^{2}$ (Lightly-loaded)

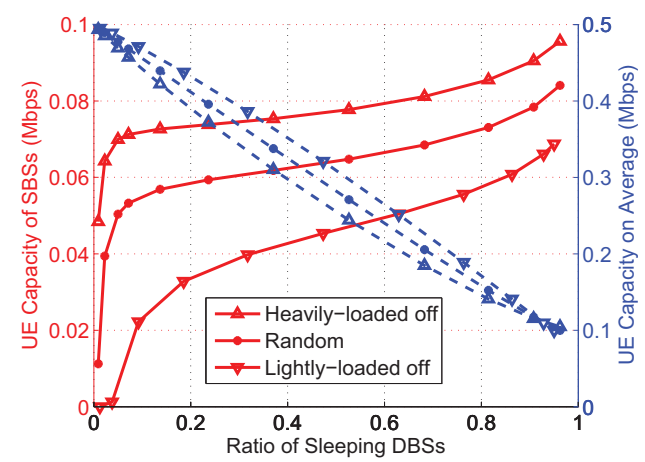

(b) $\lambda=2500 / \mathrm{km}^{2}$ (Overloaded)

Fig. 4: Average data rate of SBS UEs under different schemes

Therefore, this result is consistent with the tradeoff relationship. As the sleeping probability increases, the energy efficiency increase and the spectrum efficiency decreases from the perspective of the whole network. Furthermore, the three dashed lines also validates the analysis in Sec. IV. The DBS sleeping scheme which provides the maximal spectrum efficiency is the optimal solution of problem (8).

\section{B. Energy Saving Performance}

To evaluate the energy saving capability of the optimal solution, we consider the temporal variation of traffic load. A typical daily traffic model shown in Fig. 5 is adopted, whose 


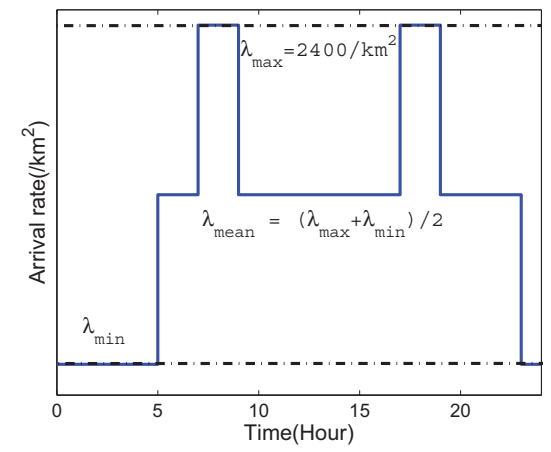

Fig. 5: Model for daily wireless traffic

two peaks correspond to the busy hours. The maximal user density $\lambda_{\max }$ is set as $2400 / \mathrm{km}^{2}$, which is the network capacity. Meanwhile, the minimal user density $\lambda_{\min }$ varies within $1 \sim 1000 / \mathrm{km}^{2}$, reflecting the dynamics of traffic load in the time domain.

Assume the total power consumption of an active BS $P_{\mathrm{BS}}$ is given by [11]:

$$
P_{\mathrm{BS}}=P_{0}+\Delta \cdot P_{\mathrm{t}},
$$

where $P_{0}$ is the static power and $P_{\mathrm{t}}$ is the transmit power of the BS, and $\Delta$ is a system parameter. The parameters for SBSs and DBSs are listed in Table I, with the subscript "s" for SBS and "d" for DBS. The power consumption of DBSs in sleep model is neglected. Numerical results are shown in Fig. 6, where the network power consumption is normalized by the power consumed without DBS sleeping.

Without traffic offloading, only the idle DBSs ${ }^{4}$ are turned off, and the energy saving gain is quite low. The reason is that the probability that a DBS goes into idle state is quite small even during the low traffic hours. Traffic offloading significantly reduces energy consumption, especially for small $\lambda_{\min }$. When the offloaded UEs requires equivalent rate as the DBS UEs, nearly $30 \%$ energy can be saved under the optimal solution when $\lambda_{\min } \rightarrow 0$. In fact, smaller $\lambda_{\min }$ means more dynamic traffic load. Therefore, the temporal variation of traffic load offers more opportunity for energy saving through DBS sleeping. Furthermore, up to $40 \%$ energy can be saved if the offloaded UEs are provided with $50 \%$ lower rate of the DBS UEs. But the spectrum efficiency is sacrificed.

\section{CONCLUSION}

In this paper, a probabilistic sleeping scheme is proposed and optimized under the separation architecture, where each DBS goes into sleep with a probability determined by its traffic load. The optimal DBS sleeping scheme which maximizes the spatial spectrum efficiency for the given power consumption is obtained. Either the lightly-loaded or the heavily-loaded DBSs should be turned off based on the ratio of DBSs turned off.

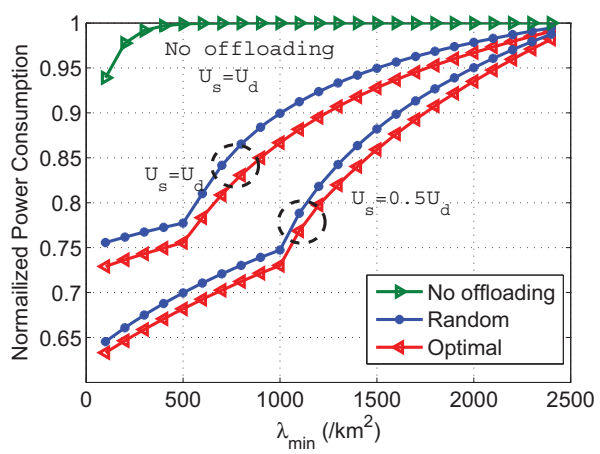

Fig. 6: Energy saving performance of optimal solution

With the optimal DBS sleeping scheme, the network power consumption can be reduced by nearly $30 \%$ under a typical daily traffic profile. In addition, more energy can be saved if the offloaded UEs can bear a lower data rate. In general, the network capacity of the optimal scheme degrades when more DBSs are turned off, which shows the tradeoff between spectrum efficiency and energy efficiency from the view of the whole network. Future works should consider non-uniform distributed traffic load and multiple service cases.

\section{REFERENCES}

[1] L. Suarez, L. Nuaymi, and J. Bonnin, "An overview and classification of research approaches in green wireless networks," EURASIP J. Wireless Commun. and Netw., Special Issue: Green Radio, Apr., 2012.

[2] Z. Niu, "TANGO: traffic-aware network planning and green operation", IEEE Wireless Commun., vol. 18, pp. 22-29, Oct. 2011.

[3] Z. Niu, et al., "Energy efficiency and resource optimized hypercellular mobile comminication system architecture and its technical challenges," Scientia Sinica(Informationis), vol. 42, no. 10, pp. 11911203, 2012.

[4] A. Capone, A. F. dos Santos, I. Filippini, and B. Gloss, "Looking beyond green cellular networks," in IEEE WONS'12, Courmayeur, Italy, Jan. 2012.

[5] X. Xu, G. He, S. Zhang, Y. Chen, and S. Xu, "On functionality separation for green mobile networks: concept study over LTE," IEEE Commun. Mag., vol. 51, no. 5, pp. 82-90, 2013.

[6] S. Zhou, J. Gong, Z. Yang, Z. Niu and P. Yang, "Green mobile access network with dynamic base station energy saving," in ACM MobiCom'09, Beijing, China, Sept. 2009.

[7] Z. Niu, Y. Wu, J. Gong, and Z. Yang, "Cell zooming for cost-efficient green cellular networks," IEEE Commun. Mag., vol. 48, no. 11, pp. 7479, Nov. 2010

[8] Y. Soh, T. quek, M. Kountouris and H. Shin, "Energy efficient heterogeneous cellular networks," IEEE J. Sel. Areas Commun., vol. 31, no. 5, pp. 840-850, May, 2013.

[9] J. Wu, S. Zhou, Z. Niu, C. Liu, P. Yang, and G. Miao, "Traffic-aware data and signaling resource management for green cellular networks," in IEEE ICC'14, Sydney, Australia, 2014 (accepted).

[10] J. Wu, S. Zhou, and Z. Niu, " Traffic-aware base station sleeping control and power matching for energy-delay tradeoffs in green cellular networks," IEEE Trans. Wireless Commun., vol. 12, no. 8, pp. 41964209, Aug. 2013.

[11] G. Auer, et al., "D2.3: energy efficiency analysis of the reference systems, areas of improvements and target breakdown," INFSO-ICT247733 EARTH, Tech. Rep., Nov., 2010. [Online]. Available: www.ictearth.eu/publications/ deliverables/deliverables.html. 